\title{
Use of Silodosin to Visualize the Posterior Urethra in Pelvic Floor Urethral Distraction Defect Patients
}

\author{
Nikhil Ranjan ${ }^{1,}$; Rana Pratap Singh ${ }^{1}$; Ahsan Ahmed ${ }^{1}$; Vijoy Kumar ${ }^{1}$; Mahendra Singh ${ }^{1}$ \\ ${ }^{1}$ Department of Urology, Indira Gandhi Institute of Medical Sciences, Bihar, India \\ ${ }^{*}$ Corresponding author: Nikhil Ranjan, Department of Urology, Indira Gandhi Institute of Medical Sciences, Bihar, India. Tel: +91-9939219883, E-mail: nikhil599@yahoo.com \\ Received: February 23, 2015; Accepted: June 13, 2015
}

\begin{abstract}
Background: Retrograde urethrogram and voiding cystourethrogram are used to define length and location of urethral stricture prior to surgery. We used a single dose of silodosin prior to VCUG to relax the bladder neck and achieve visualization of posterior urethra.

Objectives: To evaluate the efficacy of silodosin in visualization of posterior urethra during VCUG, and to compare the findings with a control group.

Patients and Methods: Patients were divided into two groups Aand B containing 20 and 15 patients, respectively. Patients in group A were given a single dose of silodosin prior to radiological studies.

Results: In group A 19 out of 20 patients were able to achieve satisfactory bladder neck opening while in group B 10 out of 15 patients were able to achieve bladder neck opening.

Conclusions: Silodosin use prior to VCUG confers a statistically significant increase in bladder neck opening and visualization of posterior urethra.
\end{abstract}

Keywords: Silodosin; Urinary Bladder Neck Obstruction; Vcug; Mcu; Urethral Stricture; Retrograde Urethrogram; Voiding Cystourethrogram

\section{Background}

Retrograde urethrogram (RGU) and voiding cystourethrogram(VCUG)are used to define the length and location of urethral stricture prior to surgery. In patients with pelvic fracture urethral distraction defect (PFUDD), the bladder neck sometimes fails to open on VCUG, leading to erroneous estimation of the stricture length. These patients are often on prolonged suprapubic diversion, which leads to decreased bladder capacity and inability to open the bladder neck. Silodosin is an alpha-1A-adrenoceptor selective antagonist that has been shown to increase urinary flow rates within 2 - 6 hours (1). We used a single dose of silodo$\sin$ (8 mg tablet) before VCUG to relax the bladder neck and achieve excellent visualization of the posterior urethra.

\section{Objectives}

Our objective was to evaluate the efficacy of silodosin in improving visualisation of posterior urethra during VCUG and compare our results with a control group.

\section{Patients and Methods}

This study was carried out in a prospective manner from January 2013 to July 2013 among patients with post-traumatic urethral stricture on suprapubic cystostomy who at- tended the urology outpatient department. Patients with partial disruption of the urethra (passing urine per urethra), straddle injury, anterior urethra stricture, and severe cardiac, hepatic, or renal disease were excluded from the study. The patients were divided into group A and group B containing 20 and 15 patients, respectively. The patients in group A were given a single dose of silodosin ( $8 \mathrm{mg}$ ) 3 hours prior to radiological studies. RGU and VCUG were performed in the radiology suite with antiseptic precautions with the patient in an oblique position. A 5-Fr infant feeding tube was placed in the fossa navicularis, and $15-20 \mathrm{~mL}$ of contrast (Meglumine Iothalamate, TAZOGRAF) was injected for the RGU.VCUG was obtained after filling the bladder via the suprapubic tube. About $300-400 \mathrm{~mL}$ of diluted contrast (60:40 contrast: normal saline) was used. Images were obtained using fluoroscopy or static radiographs.

\section{Results}

Thirty-five men, divided into group A $(n=20)$ and group $B(n=15)$, were evaluated during the study period. All the patients had a suprapubic catheter in situ for at least 3 months. The average age of the patients was 29.1 years $($ range $=22-45)$ in group A and 32.4 years $($ range $=20-41)$ 
in group B. In group A, 19 out of the 20 patients were able to achieve satisfactory opening of the bladder neck, while in group B, 10 out of the 15 patients were able to open the bladder neck. The results were analyzed using a paired ttest ( $95 \%$ confidence interval, $\mathrm{P}=0.027$ ). No patient reported any adverse effects after the single dose of silodosin.

\section{Discussion}

A combined RGU and VCUG has remained the bedrock of the diagnosis of urethral stricture, although both magnetic resonance urethrography and urethral ultrasound have been shown to be superior (2). A poorly performed urethrogram leads to an incorrect estimation of the stricture length (3). Sometimes the bladder neck fails to open on VCUG, which leads to an erroneous estimation of the stricture length. The evaluation of the posterior urethra is extremely important in PFUDD patients as this a critical factor determining the success of surgical procedures. The disruption of urethral continuity as well as the presence of hematoma and spongiofibrosis in the surrounding tissues can give rise to the wide separation of the urethral ends. Although a static cystogram has been regarded as a sign of an intact bladder neck, this finding can be misleading. These patients are often on prolonged suprapubic diversion, which results in decreased bladder capacity and inability to tolerate sufficient bladder distension to open the bladder neck voluntarily. A technique of passing a curved urethral sound antegrade from the suprapubic tract into the posterior urethra has been described to identify the proximal limit of stricture. However, this can cause bleeding and bladder neck injury (4).

Magnetic resonance urethrography has been used to evaluate obliterative posterior urethral stricture. It uses $\mathrm{T} 1$ and T2 echo-weighed spin sequences after the distension of the urethra with a sterile lubricating jelly. It has been reported to produce excellent images of urethral stricture along with spongiofibrosis. Its disadvantages include its limited availability and the high cost of the investigation (4).

Sonourethrography has also been used to evaluate the urethra. The examination is performed after the instillation of sterile saline into the urethra using 7.5-MHZ probes. The probe is placed on the ventral surface of the penis to evaluate the penile and bulbar urethra. The degree of spongiofibrosis can be assessed by noting the distensibility of the urethra. Sonourethrography provides limited imaging of the posterior urethra. Transperineal imaging has been advocated to overcome this limitation. A further limitation is the high degree of technical expertise required for the scan as most radiologists do not routinely perform this investigation (5).

The antegrade urethrogram technique has been described to visualize the proximal bulbar urethra in anterior urethra stricture patients. An optical cystoscope is passed antegrade via the suprapubic cystostomy tract into the posterior urethra, and a ureteric catheter is used to inject the contrast prior to imaging. This technique is not useful to evaluate the posterior urethra because the contrast rapidly passes into the bladder without entering the posterior urethra. Also, it requires anesthesia and an operating theatre complex (6).

Silodosin is an alpha-1A-adrenoceptor antagonist used for the treatment of the lower urinary tract symptoms associated with benign prostatic hyperplasia. It relaxes the bladder neck and prostatic urethra and has been shown to increase the urinary flow rates 2 - 6 hours after the first dose (1). It is associated with a high degree of affinity for the $1 \mathrm{~A}$ subtype of alpha-adrenoceptors, predominantly located in the prostate and lower urinary tract. Silodosin is associated with a low incidence of side effects (e.g. orthostatic hypotension and retrograde ejaculation) due to its selective action $(7,8)$. The ingestion of a single dose of silodosin led to a statistically significant increase in the bladder neck opening in our study. No adverse events were reported by our patients. Silodosin was evaluated for this function by Nagathan et al. (9) in 40 patients. They reported good bladder neck opening in 10 out of 12 patients. Often anxious patients fail to void during VCUG when the voiding command is given. This leads to multiple fluoroscopic images being obtained with increase in the radiation exposure of the patient. Silodosin circumvents these problems by opening the bladder neck and ensuring the flow of the contrast into the posterior urethra.

In our cohort of patients, silodosin use prior to VCUG conferred a statistically significant increase in the visualization of the posterior urethra and was safe with no adverse effects.

\section{Authors' Contributions}

All authors have contributed equally to data acquisition, setting objectives, planning the study, and literature search.

\section{References}

1. Marks LS, Gittelman MC, Hill LA, Volinn W, Hoel G. Rapid efficacy of the highly selective alpha(1A)-adrenoceptor antagonist silodosin in men with signs and symptoms of benign prostatic hyperplasia: pooled results of 2 phase 3 studies. J Urol. 2013;189(1 Suppl):S122-8.

2. Safwat A, Bissada N, Elgammal M, Abdalla M. 2146 Urethral Ultrasound Versus Magnetic Resonance Urethrography Versus Retrograde Urethrography in the Evaluation of Anterior Urethral Stricture: A Prospective Study. J Urology. 2010;183(4):e834.

3. Breyer BN, Cooperberg MR, McAninch JW, Master VA. Improper retrograde urethrogram technique leads to incorrect diagnosis. JUrol. 2009;182(2):716-7.

4. Sung DJ, Kim YH, Cho SB, Oh YW, Lee NJ, Kim JH, et al. Obliterative urethral stricture: MR urethrography versus conventional retrograde urethrography with voiding cystourethrography. Radiology. 2006;240(3):842-8.

5. Gong EM, Arellano CM, Chow JS, Lee RS. Sonourethrogram to manage adolescent anterior urethral stricture. J Urol. 2010;184(4 Suppl):1699-702.

6. Goel A, Gupta A, Dalela D. Antegrade urethrogram: A technique to visualize the proximal bulbous urethral segment in anterior urethral stricture. Indian J Urol. 2009;25(3):415-6.

7. Tatemichi S, Kobayashi K, Maezawa A, Kobayashi M, Yamazaki Y, Shibata N. [Alpha1-adrenoceptor subtype selectivity and organ 


\section{Ranjan $N$ et al.}

specificity of silodosin (KMD-3213)]. Yakugaku Zasshi. 2006;126 Spec no.:209-16.

8. Yamada S, Ito Y, Tsukada H. alpha1-Adrenoceptors and muscarinic receptors in voiding function - binding characteristics of therapeutic agents in relation to the pharmacokinetics. Br J Clin
Pharmacol.2011;72(2):205-17.

9. Nagathan DS, Dalela D, Sankhwar S, Goel A, Dwivedi AK, Yadav R. Single dose silodosin prior to voiding cystourethrogram: a pharmacological adjunct to enhance visualization of posterior urethra. Urol J. 2014;11(1):1320-4 